\title{
Correction to: The use of transects for resilient design: core theories and contemporary projects
}

\author{
SaMin Han (1)
}

Published online: 23 March 2021

(C) Springer Nature B.V. 2021

Correction to: Landscape Ecology https://doi.org/ 10.1007/s10980-020-01172-9

In the original publication of the article, Table 1 was incorrectly published and the correct Table 1 is given in this correction.

The original article has been corrected.

The original article can be found online at https:// doi.org/10.1007/s10980-020-01172-9.

\section{S. Han $(\bowtie)$}

Department of City and Regional Planning, University of Pennsylvania, Philadelphia, USA

e-mail: hansamin7@gmail.com; hsa@upenn.edu 
Table 1 Comparison of transect theories (created by the author)

\begin{tabular}{|c|c|c|c|c|c|}
\hline & Charles Darwin & Patrick Geddes & Ian McHarg & New Urbanism & $\begin{array}{l}\text { Landscape } \\
\text { Urbanism }\end{array}$ \\
\hline \multirow[t]{2}{*}{ Period } & 1809-1882 & $1854-1932$ & 1920-2001 & 1980s - & Mid-1990s - \\
\hline & $\begin{array}{l}\text { On the Origin of } \\
\text { Species (1859) }\end{array}$ & $\begin{array}{l}\text { Cities in Evolution } \\
(1915)\end{array}$ & $\begin{array}{l}\text { Design with Nature } \\
(1969)\end{array}$ & $\begin{array}{l}\text { Congress for the } \\
\text { New Urbanism } \\
(1993)\end{array}$ & $\begin{array}{l}\text { Constructing } \\
\text { Landscape } \\
\text { Conference (1993) }\end{array}$ \\
\hline Transect & - & Valley section & Creative fitting & $\begin{array}{l}\text { Rural-to-urban } \\
\text { continuum }\end{array}$ & $\begin{array}{l}\text { Human-environment } \\
\text { system }\end{array}$ \\
\hline Worldview & $\begin{array}{l}\text { Evolution of the } \\
\text { species }\end{array}$ & Cities in evolution & $\begin{array}{l}\text { Mutual evolution of } \\
\text { nature and the } \\
\text { human environment }\end{array}$ & $\begin{array}{l}\text { Diversity and } \\
\text { complexity }\end{array}$ & $\begin{array}{l}\text { Remedial capacity of } \\
\text { nature; Changing } \\
\text { process over time }\end{array}$ \\
\hline $\begin{array}{l}\text { Impetus of } \\
\text { Evolution }\end{array}$ & $\begin{array}{l}\text { Competition } \\
\text { (natural selection) }\end{array}$ & Cooperation & Adaptation & $\begin{array}{l}\text { Spatial } \\
\text { appropriation }\end{array}$ & $\begin{array}{l}\text { Operation of nature } \\
\text { and adaptive capacity } \\
\text { of urban landscapes }\end{array}$ \\
\hline Method & - & $\begin{array}{l}\text { Civic survey; } \\
\text { Conservative } \\
\text { surgery }\end{array}$ & $\begin{array}{l}\text { Suitability analysis; } \\
\text { Map overlay; } \\
\text { Ecological } \\
\text { inventory }\end{array}$ & Smart code model & $\begin{array}{l}\text { Multi-scaled, } \\
\text { transdisciplinary } \\
\text { design }\end{array}$ \\
\hline $\begin{array}{l}\text { Planning Field } \\
\text { / Theorists }\end{array}$ & - & Regional planning & $\begin{array}{l}\text { Ecological } \\
\text { planning }\end{array}$ & $\begin{array}{l}\text { Andrès Duany, } \\
\text { Emily Talen, } \\
\text { Elizabeth Plater- } \\
\text { Zyberg }\end{array}$ & $\begin{array}{l}\text { Charles Waldheim, } \\
\text { James Corner, Stan } \\
\text { Allen, Frederick } \\
\text { Steiner }\end{array}$ \\
\hline \multirow[t]{2}{*}{$\begin{array}{l}\text { Relationship } \\
\text { between } \\
\text { Nature \& } \\
\text { Humans }\end{array}$} & $\begin{array}{l}\text { Humans control } \\
\text { nature }\end{array}$ & $\begin{array}{l}\text { Nature as a } \\
\text { premise for } \\
\text { human settlement }\end{array}$ & $\begin{array}{l}\text { Nature leads the } \\
\text { human } \\
\text { environment }\end{array}$ & $\begin{array}{l}\text { Balance between } \\
\text { humans and } \\
\text { nature }\end{array}$ & $\begin{array}{l}\text { Synthesis of } \\
\text { human and } \\
\text { environment } \\
\text { systems } \\
\end{array}$ \\
\hline & Human & Human + Nature & Nature \&etrumants & Nature $\rightarrow$ Humans & Fat \\
\hline
\end{tabular}

Publisher's Note Springer Nature remains neutral with regard to jurisdictional claims in published maps and institutional affiliations. 